modulo $p$. In counting the number of incongruent fractions in the set (4) we must therefore consider the number of representations (5). We shall regard two representations

$$
m n^{\prime}+m^{\prime} n=p, \quad m_{1} n_{1}{ }^{\prime}+m_{1}{ }^{\prime} n_{1}=p
$$

as the same if and only if $m=m_{1}, n^{\prime}=n_{1}{ }^{\prime}, m^{\prime}=m_{1}{ }^{\prime}$, $n=n_{1}$. If $N$ is the number of representations of this type, then the relations (6) show that

$$
N=K-(p-1) \text {. }
$$

Now $K$ by definition is equal to twice the number of distinct positive irreducible fractions whose numerators and denominators are each not greater than $\sqrt{p}$. Hence*

$$
K=4(\varphi(2)+\varphi(3)+\cdots+\varphi([\sqrt{p}]))+2,
$$

where $\varphi(k)$ denotes the number of integers $<k$ and prime to it. We therefore have

Theorem III. If $p$ is a prime, then the number of representations of $p$ in the form

$$
x y+x^{\prime} y^{\prime},
$$

where $x, y, x^{\prime}, y^{\prime}$ are all positive integers $<\sqrt{p}$, is equal to

$$
-(1+p)+4 \sum_{k=1}^{[\sqrt{\bar{p}}]} \varphi(k)
$$

\title{
PROOF OF A GENERAL THEOREM ON THE LINEAR DEPENDENCE OF $p$ ANALYTIC FUNCTIONS OF A SINGLE VARIABLE.
}

BY MR. HAROLD MARSTON MORSE.

(Read before the American Mathematical Society, September 5, 1916.)

A PROOF of the following theorem has to my knowledge not been published to date. The theorem contains as a special case the ordinary theorem concerning the wronskian. Its usefulness in a general treatment of single-valued func-

* Lucas, Théorie des Nombres, p. 393. 
tions on a Riemann surface by means of abelian integrals of the second kind was pointed out by Professor Osgood in a lecture course just completed at Harvard.

Let there be given $p$ functions, $f_{1}(t), f_{2}(t), \cdots, f_{p}(t)$, analytic in a region $S$ of the $t$-plane. Consider the $p$-square functional determinant, the $i$ th row of which $(i=1,2, \cdots, p)$ is

$$
\begin{array}{r}
f_{i}^{\left(\lambda_{1}\right)}\left(t_{1}\right), f_{i}{ }^{\left(\lambda_{1}-1\right)}\left(t_{1}\right), \\
\cdots, f_{i}\left(t_{1}\right), f_{i}^{\left(\lambda_{2}\right)}\left(t_{2}\right), f_{i}{ }^{\left(\lambda_{2}-1\right)}\left(t_{2}\right), \cdots, f_{i}\left(t_{2}\right), \\
\cdots, \cdots, f_{i}{ }^{\left(\lambda_{\mu}\right)}\left(t_{\mu}\right), f_{i}{ }^{\left(\lambda_{\mu}-1\right)}\left(t_{\mu}\right), \cdots, f_{i}\left(t_{\mu}\right) .
\end{array}
$$

We denote this determinant by $D\left[t_{1}, t_{2}, \cdots, t_{\mu}\right]$. We shall have occasion to indicate determinants of the type of $D$ by enclosing the $i$ th row, without the subscript $i$, in two vertical bars.

Theorem. A necessary and sufficient condition for the linear dependence of $f_{1}, f_{2}, \cdots, f_{p}$ is that $D$ vanish identically in all of its arguments.

Consider a determinant $\bar{D}\left[\bar{t}_{1}, t_{1}, t_{2}, \cdots, t_{\mu}\right]$, obtained from $D$ by replacing the first column of $D$ by a column $f_{1}(\bar{t}), f_{2}(\bar{t})$, $\cdots, f_{p}(\bar{t})$, where $\bar{t}$ is a variable independent of $t_{1}, t_{2}, \cdots, t_{\mu}$. We will first show that $\bar{D} \equiv 0$ in all of its arguments, if the same is true for $D$. If $\lambda_{1}=0$, we have immediately that

$$
\bar{D}\left[\bar{t}, t_{1}, t_{2}, \cdots, t_{\mu}\right] \equiv D\left[\bar{t}, t_{2}, \cdots, t_{\mu}^{\dagger}\right] \equiv 0 .
$$

If $\lambda_{1}>0$, we will prove that $\bar{D} \equiv 0$ by showing that at any point $t_{1}, t_{2}, \cdots, t_{\mu}$, and for $\bar{t}=t_{1}$

$$
\begin{aligned}
& \frac{\partial^{n}}{\partial \bar{t}^{n}} \bar{D}=\mid f^{(n)}\left(t_{1}\right), f^{\left(\lambda_{1}-1\right)}\left(t_{1}\right), f^{\left(\lambda_{1}-2\right)}\left(t_{1}\right), \cdots, \cdots, \\
& f^{\prime}\left(t_{\mu}\right), f\left(t_{\mu}\right) \mid=0 \quad(n=1,2,3, \cdots),
\end{aligned}
$$

where the last $p-1$ columns of the determinant of (1) are the same as the corresponding columns of $D$. Equation (1) holds for $n=1$, since for $n=1$ the determinant of (1) either has two columns identical, or else is the determinant $D$. We proceed to prove by mathematical induction that (1) holds for all values of $n$. We therefore assume that (1) holds for $n=m$, that is, that

$$
\left|f^{(m)}\left(t_{1}\right), f^{\left(\lambda_{1}-1\right)}\left(t_{1}\right), f^{\left(\lambda_{1}-2\right)}\left(t_{1}\right), \cdots, \cdots, f^{\prime}\left(t_{\mu}\right), f\left(t_{\mu}\right)\right| \equiv 0
$$

in $t_{1}, t_{2}, \cdots, t_{\mu}$. Upon differentiating (2) with respect to $t_{1}$, we have 


$$
\begin{aligned}
& \left|f^{(m+1)}\left(t_{1}\right), f^{\left(\lambda_{1}-1\right)}\left(t_{1}\right), f^{\left(\lambda_{1}-2\right)}\left(t_{1}\right), \cdots, \cdots, f^{\prime}\left(t_{\mu}\right), f\left(t_{\mu}\right)\right| \\
& \quad+\left|f^{(m)}\left(t_{1}\right), f^{\left(\lambda_{1}\right)}\left(t_{1}\right), f^{\left(\lambda_{1}-2\right)}\left(t_{1}\right), \cdots, \cdots, f^{\prime}\left(t_{\mu}\right), f\left(t_{\mu}\right)\right| \equiv 0,
\end{aligned}
$$

where the last $p-1$ columns of the first determinant of (3) are the same as those of (1), and the last $p-2$ columns of the second determinant of (3) are the same as those of (1).

To prove the second determinant of (3) equal to zero, consider a matrix made up of the columns of $D$ together with the first column of the determinant of (2). We assume for the present that some cofactor $\bar{A}$ of the elements of the first column of $D$ does not vanish identically. We observe that in the matrix above there are only two $p$-rowed determinants having $\pm \bar{A}$ as a first minor, namely (2) and $D$. Whence* any $p$-rowed determinant of the given matrix vanishes. The latter determinant of (3) is such a determinant. Whence the first determinant of (3) vanishes identically. The induction is complete, and we have that

$$
\bar{D}\left[\bar{t}, t_{1}, t_{2}, \cdots, t_{\mu}\right] \equiv 0 .
$$

If $\bar{D}$ be developed with respect to the elements of its first column, and for a set of values of $t_{1}, t_{2}, \cdots, t_{\mu}$, for which the above mentioned cofactor $\bar{A} \neq 0$, we have

$$
A_{1} f_{1}(\bar{t})+A_{2} f_{2}(\bar{t})+\cdots+A_{p} f_{p}(\bar{t}) \equiv 0
$$

in $\bar{t}$, where the $A$ 's are constants with respect to $\bar{t}$, and not all zero. The proof of the theorem is complete under the assumption that some cofactor $\bar{A} \neq 0$.

In the contrary case, we take in place of $D$, and from the upper right-hand corner of $D$, the largest determinant for which not all of the cofactors of the elements of its first column vanish identically. There will be such a determinant unless all the $f$ 's vanish identically, in which latter case the $f$ 's are obviously linearly dependent. Further such a determinant will be of the same general form as $D$. It will be identically zero in all of its arguments; for if not, it could not be the largest determinant for which not all of the cofactors of

* If in a given matrix a certain $r$-rowed determinant is not zero, and all the $(r+1)$-rowed determinants of which this $r$-rowed determinant is a first minor are zero, then all the $(r+1)$-rowed determinants of the matrix are zero. Cf. Bôcher, Introduction to Higher Algebra, p. 54. 
the elements of its first column vanish identically. The proof applied to $D$ is therefore applicable to this determinant and the theorem is proved in general.

HaRVARD UNIVERSITY, June 1, 1916.

\section{NOTE ON THE LINEAR DEPENDENCE OF ANALYTIC FUNCTIONS.}

BY DR. G. A. PFEIFFER.

(Read before the American Mathematical Society, September 5, 1916.)

The theorem proved in the preceding note, that a necessary and sufficient condition that $p$ analytic functions, $f_{1}(t), f_{2}(t)$, $\cdots, f_{p}(t)$, be linearly dependent is that the determinant whose $i$ th row is

$$
\begin{gathered}
f_{i}^{\left(\lambda_{1}\right)}\left(t_{1}\right), f_{i}^{\left(\lambda_{1}-1\right)}\left(t_{1}\right), \cdots, f_{i}\left(t_{1}\right), f_{i}^{\left(\lambda_{2}\right)}\left(t_{2}\right), f_{i}^{\left(\lambda_{2}-1\right)}\left(t_{2}\right), \cdots, f_{i}\left(t_{2}\right), \\
\cdots, \cdots, f_{i}^{\left(\lambda_{\mu}\right)}\left(t_{\mu}\right), f_{i}^{\left(\lambda_{\mu}-1\right)}\left(t_{\mu}\right), \cdots, f_{i}\left(t_{\mu}\right) \\
\left(i=1,2, \cdots, p ; p=\mu+\sum_{i=1}^{\mu} \lambda_{i}\right)
\end{gathered}
$$

vanish identically in $t_{1}, t_{2}, \cdots, t_{p}$, can be readily proved if we assume the fundamental theorem that the identical vanishing of the wronskian of $p$ analytic functions implies their linear dependence.

By rearranging the columns of the determinant of the theorem we obtain the determinant $\Delta$ whose $i$ th row is

$$
\begin{array}{r}
f_{i}\left(t_{1}\right), f_{i}{ }^{\prime}\left(t_{1}\right), \cdots, f_{i}{ }^{\left(\lambda_{1}\right)}\left(t_{1}\right), f_{i}\left(t_{2}\right), f_{i}{ }^{\prime}\left(t_{2}\right), \cdots, f_{i}^{\left(\lambda_{2}\right)}\left(t_{2}\right), \\
\cdots, \cdots, f_{i}\left(t_{\mu}\right), f_{i}{ }^{\prime}\left(t_{\mu}\right), \cdots, f_{i}{ }^{\left(\lambda_{\mu}\right)}\left(t_{\mu}\right) .
\end{array}
$$

Without losing any generality we shall assume that

$$
\lambda_{1} \geqq \lambda_{2} \geqq \cdots \geqq \lambda_{\mu} .
$$

Now the derivative of order $n q$ of the $q$-rowed determinant whose $i$ th row $(i=1,2, \cdots, q)$ is

$$
f_{i}(t), f_{i}^{\prime}(t), \cdots, f_{i}^{(q-1)}(t)
$$

is equal to a positive integer times the $q$-rowed determinant whose $i$ th row is 